\title{
Logarithmic Barrier Function Method \\ for Convex Quadratic Programming Problem
}

\author{
Xinghua Wang (Corresponding author) \\ college of Science, Yanshan University \\ Qinhuangdao 066004, China \\ E-mail: wxh811129@163.com \\ Wen Liu \\ college of Science, Yanshan University \\ Qinhuangdao 066004, China \\ Pingping Qin \& Lifeng Sun \\ college of Science, Yanshan University \\ Qinhuangdao 066004, China
}

\begin{abstract}
In this paper, we present a kind of interior point algorithm for solving Convex Quadratic Programming problem using Logarithmic Barrier Function. In which Newton Method and Interior Penalty Function Method are combined to obtain a simple construct and easily calculating algorithm. In each iterate it needs only to solve an equality constrained Quadratic Programming problem.
\end{abstract}

Keywords: Quadratic Programming, Logarithmic Barrier Function, Newton Method, Convergence

\section{Introduction}

Solution of Quadratic Programming problem is an important subject in Mathematical Programming and Industry domain, which has wide practice background. There are many algorithms for Quadratic Programming problem, such as Potential Function Projection Method; Affine Scaling Method; Primal-dual Interior Point Algorithm and so on. In this paper, we improved the Methods of document, we obtain the search direction of $d_{k}$ by Newton Method and then solve the optimal solution of equality constrained problem.

\section{Convergence}

To Nonlinear Programming problem: $(P) \min \{f(x) \mid A x=b, x \geq 0\}$

In which $f(x)=\frac{1}{2} x^{T} Q x+c^{T} x \quad c \in R^{n}, b \in R^{m}, A \in R^{n \times m}, Q \in R^{n \times m}, x \in R^{n}$

Let $\Omega_{\text {int }}=\left\{x \in R^{n} \mid A x=b, x>0\right\}, \operatorname{Rank}(A)=m, Q$ is a symmetric positive matrix.

Considering corresponding Logarithmic Barrier Function problem $\quad:\left(P_{u_{k}}\right) \min \left\{f(x)-u_{k} \sum_{i=1}^{n} \ln x_{i} \mid A x=b, x>0\right\}$

In which $u_{k}>0$ is a penalty factor.

So to the optimal solution of $(P)$ and $\left(P_{u_{k}}\right)$,we give such theorem:

Theorem 1:If $f(x)$ is an Convex Function, $\Omega_{\text {int }}=\{x \mid A x=b, x>0\} \neq \phi,\left\{u_{k}\right\}$ is a strict monotone decreasing Sequence of positive numbers which goes to zero,If we let $x^{*}$ is the optimal solution of $(P)$, 
$x\left(u_{k}\right)$ is the optimal solution of $\left(P_{u_{k}}\right)$, Then $x\left(u_{k}\right)$ is the optimal solution of $(P)$, too.

Proof:First proof $\left\{P\left(x_{u_{k}}, u_{k}\right)\right\}$ is a strict monotone decreasing Sequence with lower-bound.

Because $\quad x_{u_{k}} \in \Omega_{\text {int }}$

So

$P\left(x_{u_{k+1}}, u_{k+1}\right) \leq P\left(x_{u_{k}}, u_{k+1}\right)=f\left(x_{u_{k}}\right)+u_{k+1} B\left(x_{u_{k}}\right) \leq f\left(x_{u_{k}}\right)+u_{k} B\left(x_{u_{k}}\right)=P\left(x_{u_{k}}, u_{k}\right)$

$P\left(x_{u_{k}}, u_{k}\right)=f\left(x_{u_{k}}\right)+u_{k} B\left(x_{u_{k}}\right) \geq f\left(x_{u_{k}}\right) \geq f\left(x^{*}\right)$

and

that $\left\{P\left(x_{u_{k}}, u_{k}\right)\right\}$ is strict monotone decreasing and has a lower-bound,

So there exists limiting $\left(P_{0}\right)$, Next Proof

$P_{0}=f\left(x^{*}\right)$

We use evidence to the contrary

If $P_{0}>f\left(x^{*}\right)$, Then there must exists

$\varepsilon>0$

Such that $P_{0}>f\left(x^{*}\right)+2 \varepsilon$

We can see from the continuity of $f(x)$, there exists

$\bar{x} \in \Omega_{\text {int }}$ let $f(-\bar{x})<f\left(x^{*}\right)+\varepsilon$

Because

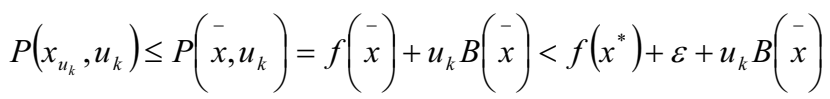

Also when $k$ is sufficiently large, there has $u_{k} B\left(\begin{array}{l}- \\ x\end{array}\right)<\varepsilon$, So we have

$\lim _{k \rightarrow \infty} P\left(x_{u_{k}}, u_{k}\right) \leq f\left(x^{*}\right)+2 \varepsilon$

That $P_{0} \leq f\left(x^{*}\right)+2 \varepsilon \quad$ it is contrary to (2)

Then proof $\forall$ limiting point $\left(x_{0}\right)$ of $x\left(u_{k}\right)$ is the optimal solution of $(P)$

Let $\lim _{k \rightarrow \infty} x_{u_{k}}=x_{0}$

Because $x_{u_{k}} \in \Omega$ int

So $x_{0} \in \Omega_{\text {int }}$

$f\left(x_{0}\right) \geq f\left(x^{*}\right)$ if $f\left(x_{0}\right)>f\left(x^{*}\right)$ and $\lim _{k \rightarrow \infty}\left[f\left(x_{u_{k}}\right)-f\left(x^{*}\right)\right]=f\left(x_{0}\right)-f\left(x^{*}\right)>0$

$P\left(x_{u_{k}}, u_{k}\right)-f\left(x^{*}\right)=f\left(x_{u_{k}}\right)-f\left(x^{*}\right)+u_{k} B\left(x_{u_{k}}\right) \geq f\left(x_{u_{k}}\right)-f\left(x^{*}\right)$

So $\lim _{k \rightarrow \infty}\left[P\left(x_{u_{k}}, u_{k}\right)-f\left(x^{*}\right) \neq 0\right] \quad P_{0} \neq f\left(x^{*}\right)$

It is contrary to (1)

So $f\left(x_{0}\right)=f\left(x^{*}\right)$

$x_{0}$ is the optimal solution of $(P)$

To the given initial inner point $x_{1}$, when the iteration step get to $k$, $x=x_{k} \in \Omega_{\text {int }}$

the search direction of problem $\left(P_{u_{k}}\right)$ can obtain by such that solution way: 
We can consider using proper Quadratic Function to approach target function $f$ in every iteration and then we construct the search direction by the direction which the iteration point points to the minimal point of Quadratic Function, let $x_{k}$ is the approximate minimal point of $f$,we take $f$ Taylor expansion at point $x_{k}$ and use its second-order approximate.

We have

$f(x) \approx f\left(x_{k}\right)+\nabla f\left(x_{k}\right)^{T}\left(x-x_{k}\right)+\frac{1}{2}\left(x-x_{k}\right)^{T} \nabla^{2} f\left(x_{k}\right)\left(x-x_{k}\right)$

Because $\nabla^{2} f\left(x_{k}\right)$ is a symmetric matrix, if we want to solve the minimal point of $f(x)$, we may let

$\nabla f(x)=0 \quad$ that $\quad$ is

$\nabla f\left(x^{*}\right)+\nabla^{2} f\left(x_{k}\right)\left(x-x_{k}\right)=0$

We take $x_{k+1}$ as the $k+1$ times of approximate of the minimal point of $f(x)$,

$x_{k+1}=x_{k}-\left[\nabla^{2} f\left(x_{k}\right)\right]^{-1} \nabla f\left(x_{k}\right)$

$d_{k}=-\left[\nabla^{2} f\left(x_{k}\right)\right]^{-1} \nabla f\left(x_{k}\right)$

\section{Related theorem}

Theorem 2 :If $d \neq 0$, then $d$ is the reduction direction of problem $\left(P_{u_{k}}\right)$

Proof: Because $\nabla^{2} f\left(x_{k}\right)$ is positive, $\left[\nabla^{2} f\left(x_{k}\right)\right]^{-1}$ is positive,

So $\nabla f\left(x_{k}\right)^{T} d_{k}=-\nabla f\left(x_{k}\right)^{T}\left[\nabla^{2} f\left(x_{k}\right)\right]^{-1} \nabla f\left(x_{k}\right)<0$

$d_{k}$ is the reduction direction of $f$ at point $x_{k}$

Now if we want to ensure the strict feasibility of iteration solution $x_{k+1}$, we must choose proper step size $\lambda_{k}$

Because $x_{k} \in \Omega_{\text {int }} \quad x_{k+1}=x_{k}+\lambda_{k} d_{k}$

$\mathrm{A}_{x_{k}}=A x_{k}+\lambda_{k} A d_{k}=A x_{k}=b$

And $x_{k+1}=x_{k}+\lambda_{k} d_{k}>0$

Let $\lambda_{k}=r \min \left\{1,-\frac{x_{k}^{i}}{d_{k}^{i}} \mid d_{k}^{i}<0\right\} r \in(0,1)$

Generally we let $r=0.995$

\section{Logarithmic Description}

Step0: give the strict initial inner point $x_{1} \in \Omega_{\text {int }}$ and tolerance $\varepsilon>0$

$u_{1} \in(0,1), \delta \in\left(0, \frac{1}{2}\right), \beta \in(0,1)$, set $k:=1$

Step1: Compute the solution $d_{k}$ of $(3)$, if $\left\|d_{k}\right\|<\varepsilon$ terminate, $x_{k}$ is the optimal solution of problem $(P)$, otherwise, go to Step2.

Step2: Compute the solution step size $\lambda_{k}$ of (4), check if it satisfies :

$P\left(x_{u_{k}}, u_{k}\right)-P\left(x_{k}+\lambda_{k} d_{k}, u_{k}\right) \geq-\delta \lambda_{k} d_{k}{ }^{T} \nabla P\left(x_{u_{k}}, u_{k}\right)$, if it satisfies, go to

Step3,otherwise, let $\lambda_{k}:=\frac{\lambda_{k}}{2}$, go to Step2.

Step3: let $x_{k+1}=x_{k}+\lambda_{k} d_{k}, u_{k+1}=\beta u_{k}$.

Step4:set $k:=k+1$, go to Step1.

\section{References}

Bai, Fusheng. \& Zhang, Liansheng. (2000). "Approximate Global Exact Barrier Method for Logarithmic Barrier Function”. OR TRANSACTIONS. 2000(3):13-18. 
Fletcher. R. “An exact penalty function for nonlinear programming with inequality”. Math Prog.5(1973). 129-150.

Pillo. G. D. \& Grippo. L. (1989). "Exact penalty function constrained optimization.SIAM.J". Control and Optimization. 27. 1333-1360.

Ruan, Xizhen. (2003). “interior point algorithm for Convex Quadratic Programming problem”. journal south-central university for nationalities..(3):72-74.

Yuan, Yaxiang. \& Sun, Wenyu. (1999).Optimization Theory and Method. Beijing: Science Press.

Zhang, Guangcheng. (2005).Computational Method for Nonlinear Optimization Beijing: CHEP. 